\title{
FRITZ MÜLLER - Für DARWIN in Brasilien
}

\author{
STEFAN SCHNECKENBURGER
}

\begin{abstract}
FRITZ MÜLLER was one of the great biologists of the 19th century. He emigrated to Brazil and lived in the german colony Blumenau where he observed nature and recognized the relation between ants and Cecropia. Müllerian mimicry is named after him. His life, publications and relations to DARWIN are described.
\end{abstract}

\section{Zusammenfassung}

FrITZ MÜller war einer der großen Biologen des 19. Jh. Er wanderte nach Brasilien aus und lebte in der deutschen Kolonie Blumenau. Durch genaue Beobachtungen erkannte er die Beziehung zwischen Ameisen und Cecropia. Er formulierte ein „Biogenetisches Grundgesetz“. Nach ihm ist die MüLLERsche Mimikry benannt. Sein Leben und Werk sowie die Beziehungen zu DARWIN werden vorgestellt.

\section{Ein Leben für die Botanik}

MÜller - MAiER - SCHUlTZE: bei uns ein abschätziger Hinweis auf Durchschnittlichkeit. Aber Müllers haben im Bereich der Biologie wirklich Bemerkenswertes geleistet. Eine der herausragendsten und unkonventionellsten Forscherpersönlichkeiten der zweiten Hälfte des 19. Jh. war JOHANN Friedrich „Fritz“ THEODOR MÜLLER (1822-1897), der bei einer beeindruckenden Publikationsliste mit knapp 250 Nummern als „Aussteiger“ in Brasilien lebte. Er ist der Entdecker der Symbiose zwischen den Ameisenbäumen (Gattung Cecropia) und ihren Schutzameisen und hat als erster das Phänomen der nach ihm benannten MüLLERschen Mimikry beschrieben. Schon früh kam er mit den Ideen DARWINs in Berührung, die er als einer von wenigen rasch in letzter Konsequenz erfasste und zu deren vehementem Vertreter und Verteidiger er wurde. Er war der ältere Bruder des Blütenökologen Hermann MÜller (1829-1883), der wesentliche Beiträge zu dieser Forschungsdisziplin geleistet hat.

\section{Ausbildung und beruflicher Werdegang}

FrITZ MÜlLER kam aus einem thüringischen Pfarrhaus, wo er durch einen liberalen und naturkundlich interessierten Vater früh mit biologischen Fragestellungen in Berührung gekommen war. Der hochbegabte Junge (MÜLLER



las später 16 Sprachen und schrieb mehrere fehlerfrei!) zog nach dem Abitur nach Erfurt, wo er in der heute noch bestehenden Apotheke des Großvaters mütterlicherseits, JOHANN BARTHOLOMÄUS TROMMSDORFF, „Zum güldenen Schwan" eine Lehre begann, die er aber nicht abschloss. Er bezog 1840 die Universität Berlin, um Naturwissenschaften zu studieren; 1845 schloss er mit einer Promotion über die regionale Blut- 




egel-Fauna ab. Intensiv hatte er sich während einer Studienphase in Greifswald mit den Krebstieren der Ostsee und ihrer Entwicklungsgeschichte beschäftigt. Einer seiner Lehrer war der berühmte und durch sein „Handbuch der Physiologie" (1833-1849) höchst einflussreiche Physiologe, Meeresbiologe und vergleichende Anatom Johannes Peter Müller (18011858). Zu JOHANNES

MÜLLERs Schülern gehören so prominente Naturwissenschaftler und Ärzte wie ERNST HAECKEL, HermanN vON Helmholtz oder Rudolph VIRCHOw. Zunächst wollte FrITZ Gymnasiallehrer werden, legte das entsprechende Examen $\mathrm{ab}$ und begann das geforderte Probejahr. Dann aber brach er ab. In dieser Zeit entfremdete er sich mehr und mehr vom christlichen Glauben. In einem Brief an seinen Bruder HERMANN schrieb er: „An die Stelle des Glaubens, dass bei Gott kein Ding unmöglich, war die auf die feste
Basis der Wissenschaft gegründete Gewissheit, „bei Gott ist kein Unding möglich“ getreten." Später wurde er zu einem scharfzüngigen und kämpferischen Atheisten.

Unter ärmlichsten Verhältnissen und nicht mehr von seiner Familie unterstützt begann er ab 1845 in Greifswald ein Zweitstudium der Medizin, das er aber ebenfalls nicht abschloss bzw. abschließen konnte: er hätte einen Eid mit der Bekräftigungsformel „So wahr mir Gott helfe!" leisten sollen.

Dazu war er unter keinen Umständen bereit. Da er sich in der Revolutionszeit 1848 exponiert hatte, konnte er auch auf kein Entgegenkommen der Behörden hoffen. Er versuchte, sich auf das Recht der Vertreter jüdischen Glaubens zu berufen und in der Eidesformel den Gottesnamen auszulassen. Dies

Abb. 2 (oben): Die Kolonie Blumenau im Jahr 1867, nach dem Kopf eines Briefbogens aus jenem Jahre.

Abb. 3 (Mitte): Unterschrift von FriTz MÜLlER. 
wurde ihm aber verweigert. Schon in seiner Studienzeit hatte er mit dem Gedanken gespielt, auszuwandern bzw. als Schiffsarzt die Welt kennen zu lernen und so die restaurative Enge Deutschlands dieser Jahre hinter sich zu lassen. Als Arzt war er aber nie tätig; später in Brasilien leistete er wohl bei Bedarf ärztliche Hilfe. Dabei bestand er aber darauf, hierfür wie ein Tagelöhner entlohnt zu werden. Zunächst nahm er eine Tätigkeit als Hauslehrer an, nicht zuletzt mit der Hoffnung auf Veränderungen durch die Revolution. Als diese nicht eintraten, beschloss er zusammen mit seinem jüngeren Bruder AUGUST (1825-1897) in die 1850 von dem deutschen Apotheker Hermann Blumenau in der südbrasilianischen Provinz Santa Catarina gegründeten Kolonie Blumenau auszuwandern. Unmittelbar vor der Überfahrt heiratete FRITZ die Tagelöhnertochter KaROLINE TÖLLNER, die ihm schon 1848 ein erstes Kind geboren hatte. Man hatte ihm deswegen vorgeworfen, er fröne der freien Liebe. Wie vieles war auch dies eine bösartige Unterstellung einer konservativen Propaganda, denn FriTZ war mit Karoline 46 Jahre lang zusammen; das Ehepaar hatte 10 Kinder. Auf einer Heirat vor der Auswanderung muss seine Frau bestanden haben und es war auch die einzige Konzession, die er an die Kirche machen sollte. 1852 verließen die beiden Brüder mit ihren Familien Deutschland, um nie wieder dorthin zurückzukehren.

\section{Müllers Leben in Brasilien}

In Brasilien führte FRITZ zunächst das harte und gefährliche Leben eines Kolonisten: er rodete Land, machte es urbar und lebte vom Ertrag seiner Pflanzungen. Ein spartanisches und entbehrungsreiches Leben führte er im Grunde genommen bis zu seinem Tod 1897. Zunächst aber wurde er auf Betreiben Hermann Blumenaus nach Desterro (heute Florianópolis) auf der Insel Santa Catarina „weggelobt“. Der Freidenker MüLLER passte nicht so recht in die Kolonie, wo man die Religion als einen wichtigen Faktor zur Aufrechterhaltung der Ordnung und der Disziplinierung sah, zumal die staatliche Ordnungsmacht im rauen „Outback“ damals kaum präsent war. FriTz MÜLler übernahm 1856 die Stelle eines Lehrers der Mathematik und Naturwissenschaften an der dortigen Provinzschule, die er bis 1867 versah: sein Ende in Desterro fiel - wen wundert's - mit der Übernahme der Schule durch den Jesuitenorden zusammen. Der Name Desterro - zu deutsch „Verbannung“ - war es denn auch, den ERNST HAECKEL in einem Nachruf wählte, um MÜLLER von anderen Trägern des gleichen Namens zu unterscheiden: sein Bruder HERMANN (1829-1883) wurde zu „MÜLLERLippstadt“, er zu „MÜLLER-Desterro“ und sein Halbbruder WiLheLM (1857-1940), Professor in Greifswald, zum „Ostracoden (=Muschelkrebs)MÜLLER". Auch der Namensvetter und Weinrebenzüchter HERMANN MÜLLER (1850-1927) war Zeitgenosse - ihn kennt man nach seinem Geburtsort als „MÜLLER-Thurgau“. In dieser Zeit wurde FrITZ mit DARWINs Hauptwerk „Über den Ursprung der Arten“ bekannt, das er gründlich studierte und rezipierte. Er empfahl es u. a. auch seinem jüngeren Bruder HERMANN, den er wiederholt, aber ohne Erfolg, zum Auswandern drängte, und stellte später befriedigt fest, dass sich auch dieser zur Lehre DARWINs bekehrt hatte.

Die Flachwasserzonen um die Insel Santa Catarina mit ihrer überaus reichen Crustaceenfauna brachten ihn dazu, sich wie schon in Greifswald wieder mit Krebstieren zu beschäftigen Er studierte vor allem die komplizierte Individualentwicklung (Ontogenese) dieser Tiere und verfasste darüber und die sich hieraus ergebenden stam-

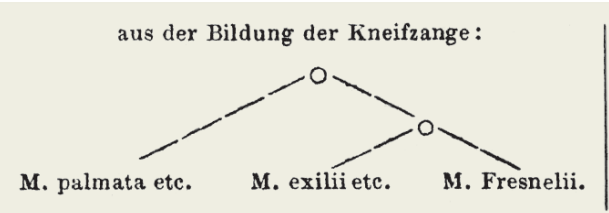

aus der An- und Abwesenheit der Nebengeissel :

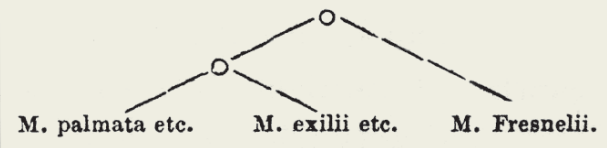

Abb. 4: Die ersten biologischen Cladogramme, entwickelt von FrITZ MÜLLER. 
mesgeschichtlichen (phylogenetischen) Konsequenzen sein einziges Buch „Für DARWIN“ (1864). Wie viele seiner Veröffentlichungen, deren Schicksal ihm stets erstaunlich gleichgültig war, ging das Manuskript an seinen Bruder HermanN, der sich um den Druck kümmerte. Das wirtschaftlich erfolglose Buch wurde auf Betreiben DARWINs ins Englische übersetzt und erschien 1869 unter dem etwas milderen Titel „Facts and Arguments for DARWIN". Hier formulierte MÜLLER als erster das später von ERNST HAECKEL aufgegriffene „Biogenetische Grundgesetz“ („In der Ontogenie wird die Phylogenie wiederholt") - allerdings in einer wesentlich präziseren und angemesseneren Formulierung: MÜLLER stellte fest, dass in der Individualentwicklung nicht Adultstadien phylogenetisch älterer Gruppen, sondern deren Ontogenesephasen wiederholt werden (können). Daneben entwarf er das erste Cladogramm der Biologie (eine Darstellung, die in dieser Form erst wieder von HeNNIG in den fünfziger Jahren des 20. Jh. aufgegriffen wurde) und untersuchte dabei die Gewichtung von Merkmalen: je nachdem, welches Merkmal er innerhalb der Krebsgattung Melita stärker bewertete, ergab sich ein anderer phylogenetischer (Stamm)Baum.

\section{MüLLER als Forscher}

Müller musste die Schule nach dem Einzug der Jesuiten 1867 verlassen. Da er aber unkündbar war, machte er den Vorschlag, für die Provinzregierung als naturwissenschaftlicher Forscher zu arbeiten und regelmäßig darüber zu berichten eine Tätigkeit, die er ernst nahm. Er kehrte nach Blumenau zu seiner Familie zurück und arbeitete wieder als Farmer. Doch ermöglichten ihm seine bescheidenen und oft mit sehr großer Verzögerung ausbezahlten Einkünfte aus seinem Regierungsposten die Durchführung von Forschungsarbeiten. Später wurde er „reisender Naturforscher" der Provinz (1867) und dann des Nationalmuseums in Río de Janeiro (1876). Diesen Posten verlor er 1891 im hohen Alter unter unwürdigen Umständen nach der Absetzung des liberalen und an Kunst und Wissenschaften interessierten Kaisers Dom PEDRO III. Stolz wie er war, lehnte er die ihm zahlreich aus Deutschland angebotene wirtschaftliche Hilfe von Kollegen ab. Themen seiner Berichte waren Beobachtungen zur Geographie und Geologie, zu Klima, Flora, Fauna sowie zur Agronomie. Ein intensiver brieflicher Austausch entwickelte sich zu zahlreichen Kollegen in Europa, wobei hier besonders sein Bruder Hermann, Charles DARWIN und ERNST HAECKEL zu nennen sind.

\section{MÜLLER und DARWIN}

ChARles DARWIN versorgte MÜller u. a. mit neuer - sowohl eigener als auch fremder Literatur - und trug Sorge um die Publikation von MÜllers Arbeiten, die der Deutsche, ohne jemals formal Englisch gelernt zu haben, in einer so geschliffenen Sprache verfasste, dass DARWIN sie ohne Änderungen und Korrekturen in den Druck geben konnte. In Reaktion auf ihm zugesandte DARWINsche Arbeiten oder von ihm formulierte Fragen wandelten sich MÜLLERs Forschungsgebiete. CHARLES DARWIN schrieb in einem Brief im September 1881: „I feel ashamed of myself for asking you so many things from you, and taking up so much of your valuable time, but I can assure you that I feel quite grateful!" - nicht ohne Grund nannte DARWIN MÜLlER einen „Fürsten der Beobachter“. So studierte MÜLLER Kletterpflanzen oder lieferte DARWIN zahlreiche Beispiele für seine Studien zu den „Verschiedenen Blüten bei Pflanzen der gleichen Art", worunter sich das damals gerade erst entdeckte Phänomen der Heterostylie („Verschiedengriffeligkeit") verbarg, das letztlich im Zusammenhang mit der Sicherung der Fremdbestäubung und der Vermeidung von Selbstbestäubung zu sehen ist. Neben botanischen Themen studierte MÜLLER die subtropische Tierwelt Südbrasiliens. So wies er nach, dass zwischen Ameisen der Gattung Azteca und den Ameisenbäumen (Gattung Cecropia) ein Zusammenleben zum gegenseitigen Nutzen besteht. Die Pflanzen liefern in ihren hohlen Stämmen Obdach und Nahrung (in Gestalt der in speziellen Haarfluren gebildeten, nach ihm benannten MüLLERschen Körperchen). Die räuberischen Ameisen bilden im Gegenzug eine „Schutztruppe“ der Pflanzen gegen Fraßfeinde wie Blattschneiderameisen, Epiphyten und Kletterpflanzen. Bekannt war 
damals die BATESsche Mimikry: „genießbare“ Insektenarten tarnen sich durch Verwendung der gleichen Warntracht wie ihr gefährliches bzw. ungenießbares Pendant - man denke nur an die häufig mit Wespen verwechselten, völlig harmlosen Schwebfliegen. MÜLLER wies nun nach, dass eine Reihe von giftigen bzw. ungenießbaren Schmetterlingsarten aus verschiedenen Verwandtschaftskreisen die gleiche Warntracht benutzt und so in geringerem Maß Beute von Fressfeinden wird. Dieses als „MülLERsche Mimikry“ bekannte Phänomen bezeichnet man besser als „Signalnormierung“, da eine Mimikry im engeren Sinn nicht vorliegt. Immer wieder ordnet MÜlLER seine Beobachtungen in den Rahmen der DARWINschen Evolutionstheorie ein und weist nach, dass erst diese Vorstellung die Phänomene erklären könne. Viele Themen hat er im Lauf seines langen Biologenlebens bearbeitet: immer wieder Crustaceen (auch wenn es Bewohner von Bromelientanks in den Baumkronen waren), die Biologie von Epiphyten (insbesondere auch von Orchideen), Bromelien, Feigen (Gattung Ficus) und die sie bestäubenden Feigengallwespen, Termiten oder die dufterzeugenden Organe von Schmetterlingen. Immer wieder beschäftigten ihn Fragestellungen, die DARWIN oder auch sein Bruder HERMANN an ihn herangetragen hatten. So fand er in Brasilien einen Nachtschmetterling mit einem $25 \mathrm{~cm}$ langen Rüssel, der die belächelte DARWINsche Voraussage eines solchen Tieres als Bestäuber der madegassischen Orchidee Angraecum sesquipedale viel wahrscheinlicher machte - wenige Jahre später (nach DARWINs und MüLLERs Tod) wurde sie bestätigt. Auch verdankt die Blütenbiologie den reichen Ergebnisschatz Hermanns letztlich den Fragen von Fritz, der den Jüngeren im Verlauf ihres umfangreichen Briefwechsels auf das Thema brachte. HERMANn, noch etwas unsicher, fragte nach, was denn in Detail zu tun sei und mit wenigen Fragen skizzierte FRITZ in seinem Antwortbrief 1866 das Forschungsprogramm, das sein Bruder in eineinhalb Jahrzehnten mit großem Erfolg be- und ausarbeitete.

Abb. 5: Orchideen - Zeichnungen von FrITZ MÜLLER.

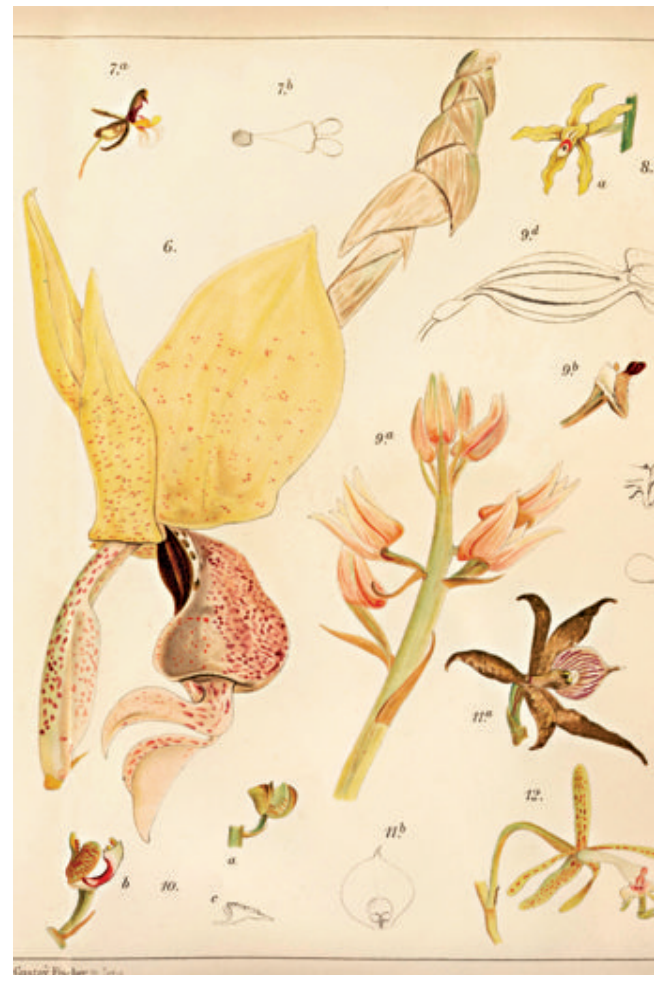

\section{Publikationen}

Müllers Publikationen erschienen in den verschiedensten Zeitschriften - von „Nature“ bis hin zum „Kosmos“, der von ERnST KRAUSE (alias CARUS STERNE; 1839-1903) herausgegebenen, ganz DARWIN und dem HaECKELschen Monismus verpflichteten „Zeitschrift für einheitliche Weltanschauung auf Grund der Entwicklungslehre in Verbindung mit Charles DarWIN und ERNST HAECKEL“ - einer im besten Sinne populärwissenschaftlichen, leider nur wenige Jahre erscheinenden Zeitschrift von hohem Anspruch. Alfred Möller, eine Neffe Müllers, hat in verdienstvoller Weise die Arbeiten seines Onkels gesammelt; die insgesamt fünfbändige Ausgabe erschien zwischen 1915 und 1921 unter dem Titel „Werke, Briefe und Leben“ im Verlag G. FISCHER (derzeit erscheint ein Nachdruck bei „Bibliolife"). Allein die beiden Textbände der veröffentlichten Werke umfassen 1500 Seiten! Daneben findet sich eine Unzahl von kleineren Beobachtungen in den Werken seiner Korrespondenzpartner wie DARWIN oder seines Bruders 
sowie in den Briefen. Nicht selten kann man bei als besondere Neuheiten gepriesenen Entdeckungen abwinken: das hat FRITZ MÜLLER vor über 100 Jahren schon gesehen und beschrieben eben leider vielfach in Briefen oder wenig beachteten Publikationen. Geehrt wurde FrITZ durch Ehrenmitgliedschaften in wissenschaftlichen Gesellschaften (so der Leopoldina oder der Senckenbergischen Naturforschenden Gesellschaft) und mehrere Ehrendoktorate (z.B. erhielt er am 4. August 1868 ein Ehrendoktorat der Universität Bonn - am gleichen Tag wie CHARLES DARWIN, LOUIS PASTEUR, der Botaniker Julius SACHS, der Chemiker Friedrich August KeKUlé und der spätere deutsche Kaiser FrIEDRICH III. Allerdings, und das charakterisiert diese


außergewöhnlich bescheidene Wissenschaftlerpersönlichkeit, hat er diese Ehrungen schnell vergessen: von seinem Neffen, dem bereits erwähnten ALFRED MöLlER darauf angesprochen, konnte er nicht einmal genau sagen, welche Universitäten ihn geehrt hatten - angesichts des heutigen Titelkaufs und Titelhandels eine schon damals seinen Neffen befremdende Haltung.

\section{MÜLleRs Domizil}

MÜLlERs Heim in Blumenau war wie in anderen Fällen von im Ausland lebenden Naturforschern oder an Naturforschung interessierten Auslandsdeutschen oft Ziel und Basis von Forschungsreisenden. So forschten 1886/87 die jungen Bonner Botaniker HeINRICH SCHENCK und ANDREAS SCHIMPER (später Mitautoren der ersten Auflagen des „STRASBURGER“, des altbewährten Lehrbuchs der Botanik) in Südbrasilien. Eines ihrer Erinnerungsfotos wurde später in der „Gartenlaube“ veröffentlicht. Es zeigt FRITZ MÜlLER in dem ihm üblichen Exkursionsanzug: barfüßig (!), mit Schlapphut, Sammeltasche und mit einem Hakenstock zum Herabholen von Epiphyten. In seinem bescheidenen Domizil verfügte er über eine kleine Handbibliothek, ein Mikroskop, Präparierbesteck und Zeichenmaterial sowie einfache Messinstrumente - und ein gigantisches Gedächtnis. Allein damit und mit seiner phänomenalen Beobachtungs- und Kombinationsgabe schuf er sein grandioses Werk.

MÜLLER starb 1897 in Blumenau hoch angesehen - über lange Jahre hinweg versah er das Amt eines Friedensrichters und kümmerte sich um die Bildungsangelegenheiten in der Kolonienach mehreren Schicksalsschlägen wie dem Tod sowohl seiner Ehefrau als seiner Lieblingstochter. Sein Grab existiert noch heute, in Blumenau gibt es ein kleines Museum sowie ein Denkmal und dort wie in seiner Heimat in Thüringen erinnern Straßen an den „Fürsten der Beobachter“, an einen der ganz großen Biologen des 19. Jahrhunderts.

Abb. 6 (oben): Fritz Müllers Besitz, Garten mit Wohnhaus 1891.

Abb. 7 (unten): Aufnahme von A. Möller: FRITZ MÜLlER in „Exkursionsanzug“. 